\title{
A Comparative Study between Traditional Learning and Virtual Learning (Design a Virtual Learning System)
}

Maisireem A. Al-Sayeg

College of Computer Science and Mathematics

University of Mosul, Iraq

\section{Received on: 25/09/2008}

\section{ABSTRACT}

Accepted on: 04/12/2008

Virtual learning is an essential source of growth in scientific in recent years by standing in the basis of many of the virtual learning programs. In this research virtual learning program has been designed (geographical for First primary school) as the first building block in opening electronic and virtual schools in Iraq to play a role in changing the mechanisms of education and teaching methods, which facilitated the use of modern techniques of voice and image programs and databases to provide classes in School anywhere in the world, for a study out of school is necessary in the educational process.

Identification has been done to determine the importance of virtual learning on 25 teachers on the subject of geographical and 100 students from first primary school of different schools in the city of Mosul, the results showed the importance of the Internet and computer and virtual learning in the development of learning process.

Keywords: mechanisms of education, teaching methods, virtual learning,

$$
\begin{aligned}
& \text { دراسة مقارنة بين التعليم التقليدي والتعليم الافتراضي (تصميم نظام تعليمي افتراضي) } \\
& \text { ميس الريم عضيد الصائغ }
\end{aligned}
$$

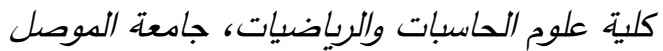

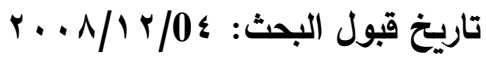

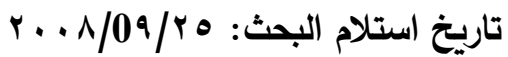

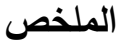

$$
\begin{aligned}
& \text { يمثل التعليم الافتراضي وما رافقه من تقدم علمي وتثني مصدرا أساسيا من مصادر النمو العلمي و ما } \\
& \text { وصلت إليه التقنية في السنوات الأخيرة من مكانة تقوم عليها بثكل أساس العديد من برامج التعليم الإفتراضي. }
\end{aligned}
$$

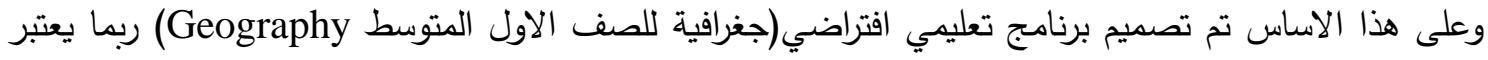

$$
\begin{aligned}
& \text { اللبنة الاولى في مجال فتح مدارس افتراضية الكترونية على مستوى العراق ليلعب دورا في تغيير آليات التعليم } \\
& \text { وطرق التدري، والذي يسر ذلك استخدام التقنيات الحديثة من برامج صوت وصورة وقواعد بيانات لتقدم الفصول } \\
& \text { الدراسية في أي مكان من العالم، ليصبح التعليم عن بعد أمرا يفرض نفسه في العملية التربوية التعليمية. }
\end{aligned}
$$

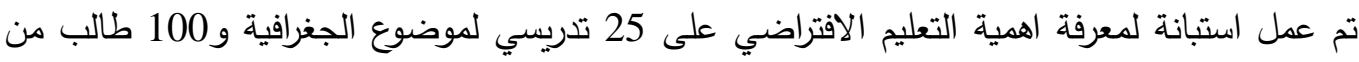

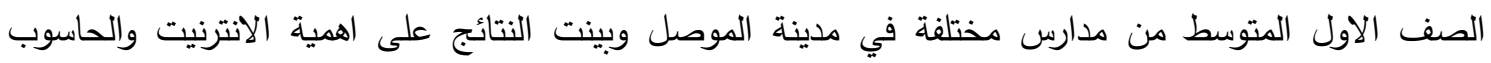

$$
\begin{aligned}
& \text { والتعليم الافتراضي في تطوير العملية التعليمية. }
\end{aligned}
$$

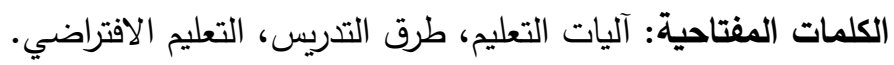

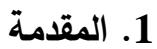

$$
\begin{aligned}
& \text { يتسم عصرنا الحالي بالتقدم العلمي والتقني الهائل والذي ساهم في إحداث كثير من التغيرات في شتى }
\end{aligned}
$$

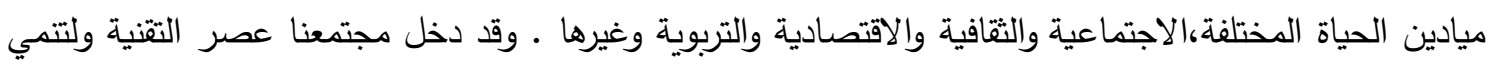

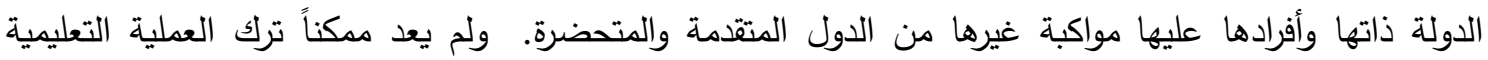

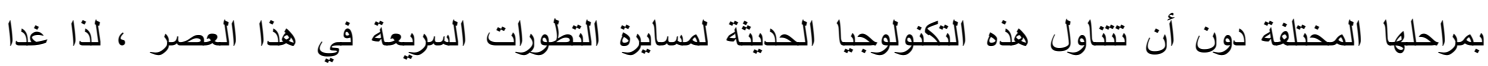


التطوير والتحديث من خلال التخطيط الجيد من أهم الأهداف التي يسعى التربويون لتحقيقها لتلبية احتياجات المجتمع ورفع مستوى نمو المتعلمين ولقد أدركت أمم كثيرة أهمية التخطيط لبناء مجتمع متقدم يكون أساسه العلم

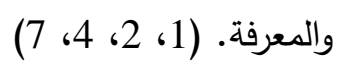

ونظرا للثورة الهائلة التي حدثت في تقنيات الاتصالات والمعلومات والتي توجت أخيراً بشبكة المعلومات الدولية (انترنت) فقد استثر التعليم هذا التقدم فظهرت الاستفادة من هذه التقنيات بتأسيس تعليم متكامل معتمد على هذه التقنيات وهو ما سمي بالتعليم الاكتروني أو الافتراضي (Virtual Learning) (1، (4).

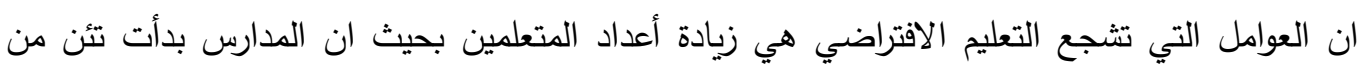

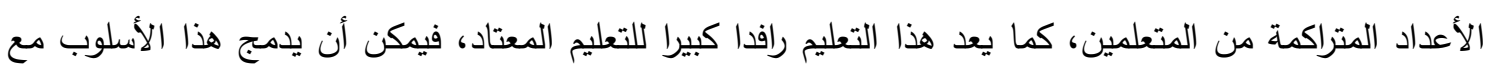

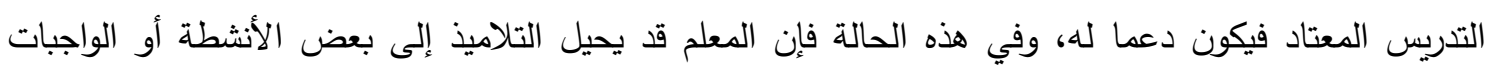

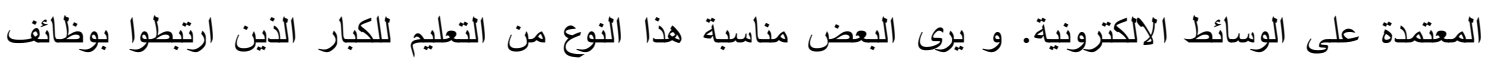

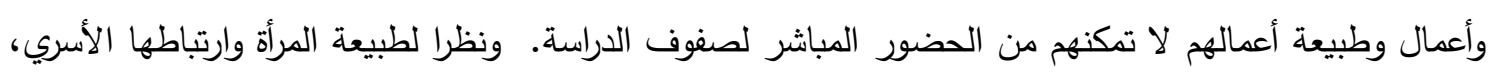

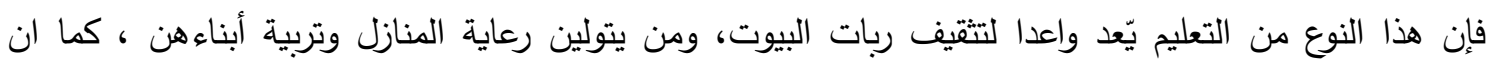

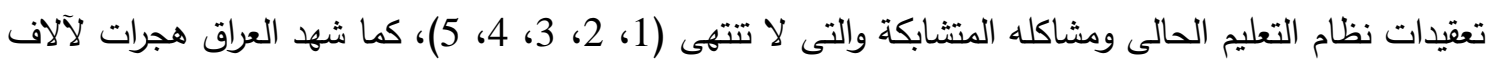
العقول العلمية العراقية في مختلف التخصصات العلمية فضلا عن توقف تدفق التقنية الحديثة إلى المجتمع العراقي بسبب الحروب والحصار وهي فترة جاوزت الثلاثة عقود أدت إلى استقرار هذه العقول في مجتمعات العالم المختلفة

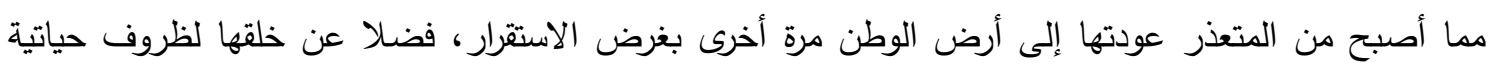

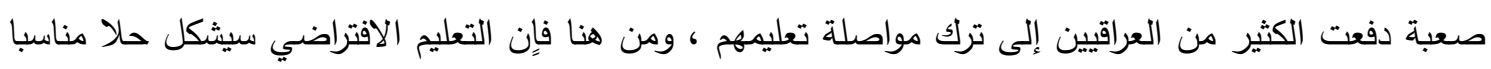

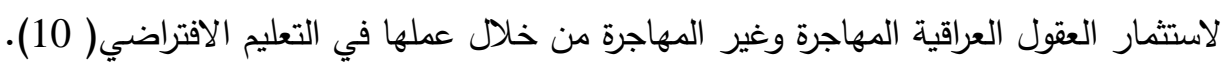

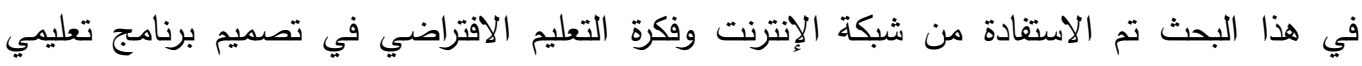
افتراضي (جغرافية للصف الاول المتوسط Geography) لتعليم منهاج الجغرافية في المدارس المتوسطة كخطوة اولى لبناء مدرسة افتراضية بهدف تأهيل الطالب إلى التفاعل مع المحيط بكفاءة وفاعلية ولمواجهة تحديات القرن الواحد والعشرين. تم في هذا البحث مناقثة المراحل التي مر بها التعليم ومنها تعريف التعليم التقليدي، التعليم باستخدام الحاسوب، التعليم الافتراضي، الاطلاع على تجارب الدول العربية والعالمية في التعليم الافتراضي

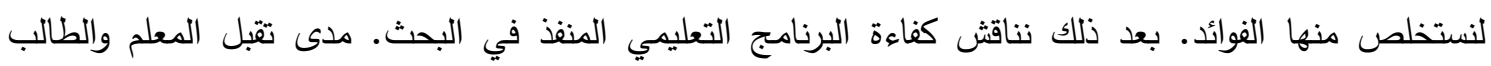

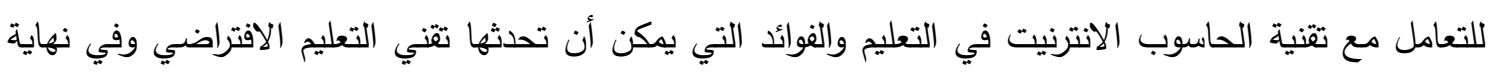
هذا البحث نخلص إلى فكرة المدرسة الافتراضية وفوائدها.

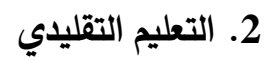

يرتكز التعليم التقليدي على ثلاثة محاور أساسية ، وهي: المعلم والمتعلّم والمعلومة.ومن أهم إيجابياته التقاء المعلم

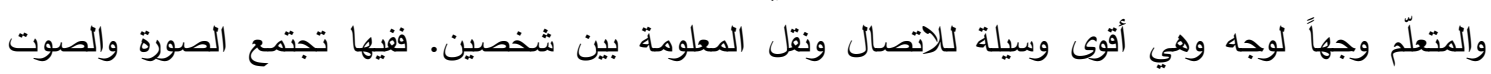
بالمشاعر والأحاسيس ، [8]. ولكن في العصر الحاضر يواجه التعليم التقليدي منفرداً بعض المشكلات مثل الزيادة

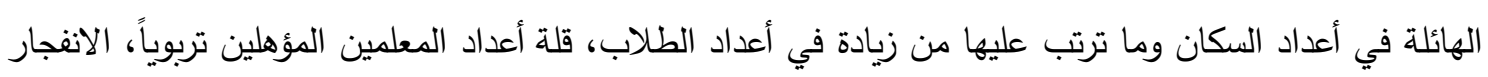
المعرفي الهائل وما ترتب عليه من تثعب في التعليم، القصور في مراعاة الفروق الفردية بين الطلاب .فالمعلم ملزم 
بإنهاء كم من المعلومات في وقت محدد مما قد لا يمكّن بعض المتعلّمين من متابعته بنفس السرعة، مع بروز مثل هذه المشكلات ، فإن الحاجة تدعو إلى استخدام وسائل تعليمية تساعد على التخفيف من آثارها.

3. التعليم باستخدام الحاسوب

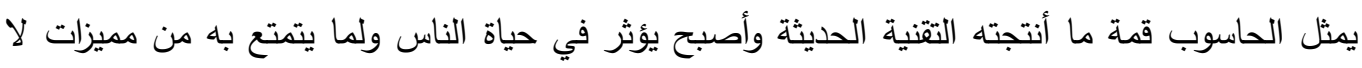
توجد في غيره من الوسائل التعليمية فقد اتسع استخدامه في العملية التعليمية. ولعل من أهم هذه المميزات: التفاعلية

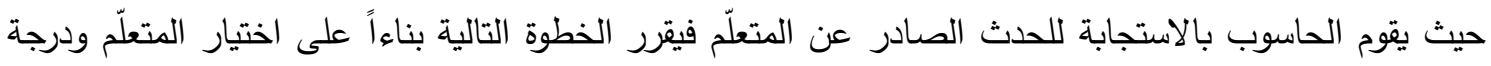

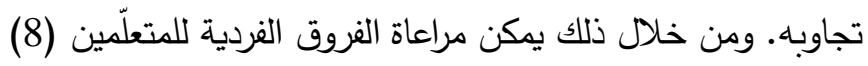

4. التعليم الافتراضي(Vertual Education)

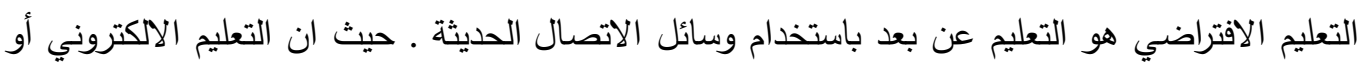

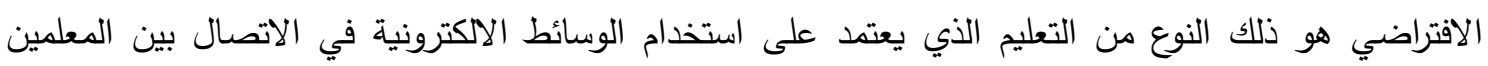

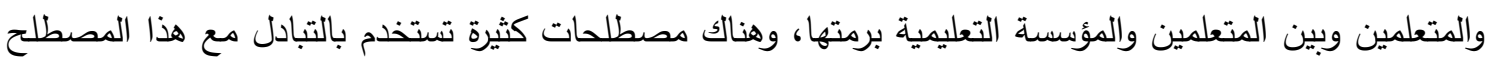

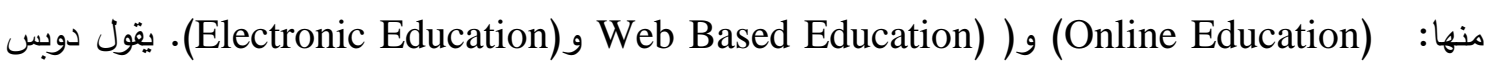
وفليب: "إن المتعلم افتراضيا (إلكترونيا) هو متعلم حقيقي لكنه يتعلم في بيئة إلكترونية".(1، 2، 4) 4.1 أدوات التعليم الافتراضي هي كل وسائل الاتصال الحديثة مثل الكمبيوتر المتصل بالانترنت ، التلفاز التفاعلي ، أجهزة الـ ( Palm) وأجهزة الـ WAP) هذه الأدوات سوف تكون في أحد الأيام من القنوات المهمة

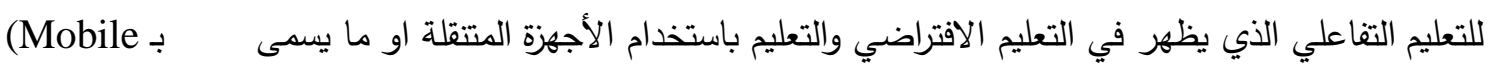
(12،11)learning )

$$
\text { ومن اهم الادوات التي يستخدمها التعليم الافتراضي هي الانترنيت وكما يلي(11، 12) }
$$
• البريد الإكتروني: استخدام البريد الإكتروني لتسهيل اتصال الطلاب فيما بينهم وتبادل المعلومات والأفكار التربوية والتواصل خارج الصف الدراسي بل والتواصل مع طلاب من دول أخرى. كذلك يستفيد المعلم من

$$
\text { البريد الإلكتروني بالتواصل مع زملائه وطلابه. }
$$

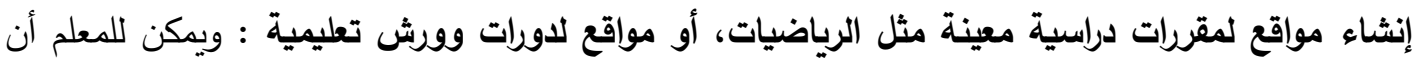
ينشئ موقع لطلابه فقط أو لطلاب البلد الذي يعيش فيه أو جميع الطلاب حول العالم ـ فالمعلم يستطيع التحكم بالموقع وتحديد المشاركين. وتقدم هذه الخدمة كذلك مجاناً. زيارة أدلة المواقع التربوية : زيارة مواقع أدلة المواقع التربوية العربية والأجنبية والتي تضم أغلب المواقع التربوية تحت موقع واحد وتسهل الوصول إلى عدد كبير من المواقع التربوية مثل دليل المواقع التربوية العربية

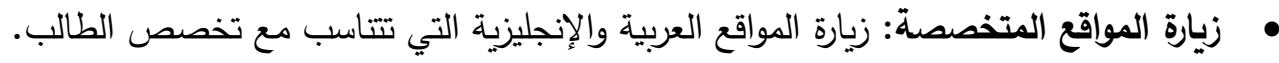
• استخدام مواقع البحث الثهيرة : مثل محركات البحث التي تقدم خدمة البحث بعدد من اللغات بما فيها اللغة العربية إنشاء المواقع الثخصية : يمكن لأي معلم أن ينشئ موقع شخصي ومن خلال الموقع يستطيع أن يتواصل مع الآخرين. 
سيحدث التعليم الافتراضي ثورة في التعليم والتدريب وهذه حقيقة مؤكدة ستحدث في كل الفصول الدراسية في العالم العربي ، فالتعليم الافتراضي سيكون هو الجيل القادم في طرق التعليم المرئية المساعدة. كما إن التعليم الافتراضي يعمل على تطوير المجتمعات باستخدام الانترنت (1،2،11،12). وهناك عوامل تشجع على هذئ هذا النوع من التعليم ومنها: زيادة أعداد المتعلمين بشكل حاد لا تستطيع المدارس المعتادة استيعابهم جميعا. قلة أعداد المعلمين المؤهلين تربوياً نظرا لطبيعة المرأة وارتباطها الأسري، فإننا نرى أن هذا النوع من التعليم يُعد واعدا لتثقيف ربات البيوت، ومن يتولين رعاية المنازل وتربية أبناءهن. وهذا النظام يُكَن الذين فاتتهم فرص التعليم بسبب العمر أو لأسباب أخرى ويساعد الموظفين وربات البيوت

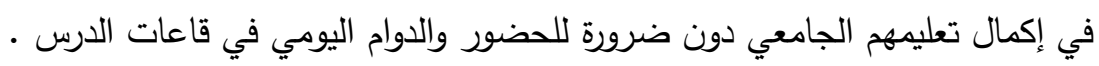
ومن إيجابياته أيضا قلة التكلفة المادية، سرعة الحصول علدئل على المعلومات، المرونة في الوقت والعمل، إعطاء التعليم صفة العالمية، تطوير مهارات الطلاب على استخدام الحاسوب، عدم التقيد بالساعات الدراسية.

4.3

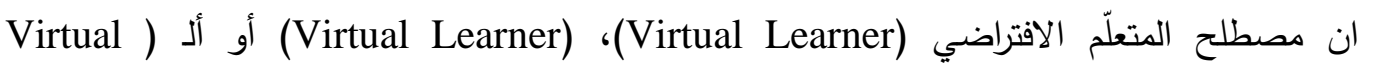
(Student

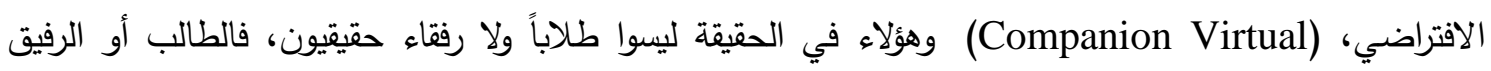

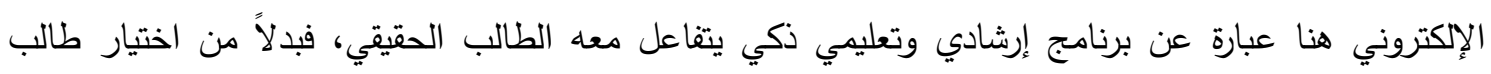

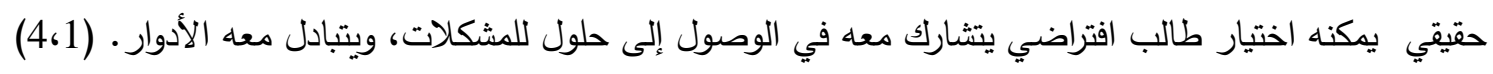

4.4

وهو المعلم الذي يتفاعل مع المتعلم افتراضيا، ويتولى أعباء الإثراف التعليمي على حسن سير التعلم، وقد

يكون هذا المعلم داخل مؤسسة تعليمية أو في منزله، وغالبا لا يرتبط هذا المعلم بوقت محدد للعمل وإنما يكون الإنتراك

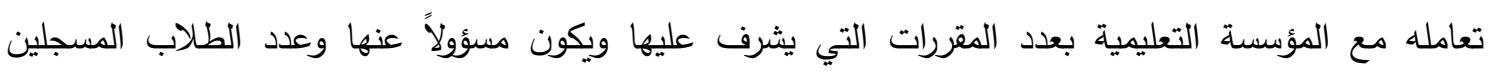
لديا(1، 4).

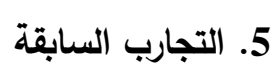

نستعرض هنا تجارب الدول العالمية والعربية في مجال الحاسوب والانترنيت في التعليم والتعليم الافتراضي ومنها :

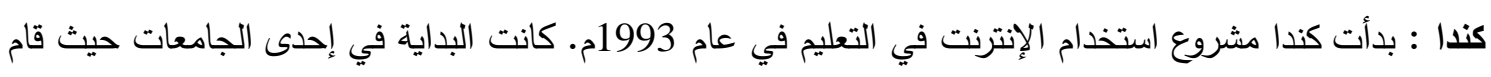

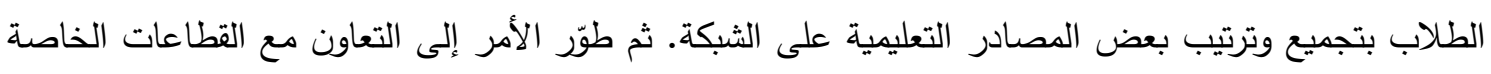

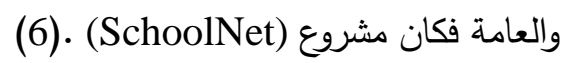
كوريا : في مارس 1996م أعلن عن بداية مشروع (KidNet) لإدخال شبكة الإنترنت في رياض الاطفال

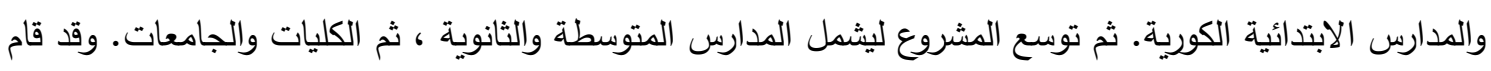
هذا المشروع من خلال التعاون بين شبكة الثباب العالمية من أجل السلام (GYN) التي نشأت في جامعة ولاية 
متشجن الأمريكية وإحدى الصحف الكورية من جانب ووزارة الاتصالات والمعلومات ووزارة التعليم الكوريتين من

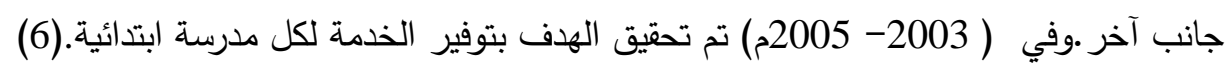

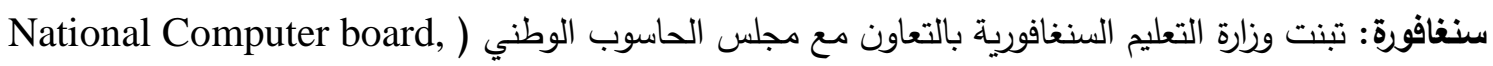
(NCB 1993م بدأ المشروع بست مدارس. وقد قادت التجربة إلى ربط المدارس والمشرفين على التعليم بالثبكة. كما تم ربط وزارة التعليم بشبكة الإنترنت. بعد ذلك توسع المشروع ليثمل الكليات المتوسطة (Junior Colleges). وقد ولد

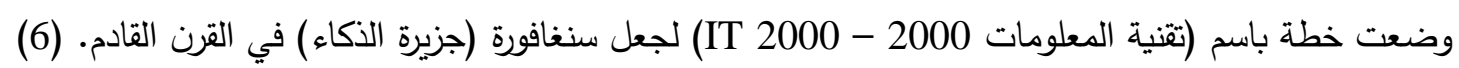
الولايات المتحدة الامريكية : مدرسة فلوريدا الافتراضية (Florida Virtual School (FVS) بدأت مدرسة فلوريدا الافتراضية Florida Virtual School أنشطتها في أغسطس 1997 كمشروع وصل بين المدرستين الإقليميتين الحكوميتين Public Schools Orange County ومدرسة Alachua County اللتين انطلقتا في فضاء الإنترنت عام 1996، وذلك بطاقة 15 تربوي خدموا في وظائف الإدارة والتعليم والتطوير • وكانت مهمة المشروع هي وضع مدرسة ثانوية كاملة عبر الإنترنت بحلول عام 2001 وتضمين الخدمات الطلابية فيها لتمكين

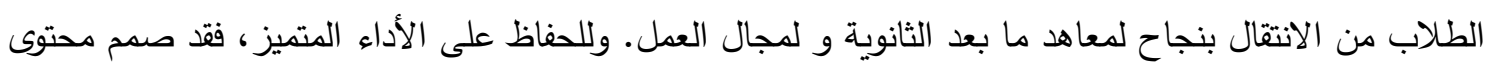

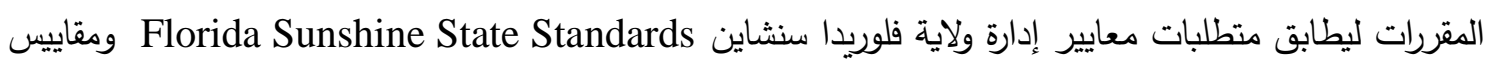

أخرى مهمة مثل مسابقات SCANS التي يدعمها كل من التعليم والمجتمعات المهنية (13). استعرضنا اعلاه تجارب الدول في العالم التي شجعت التعليم الافتراضي باستخدام الانترنيت ونستعرض تجاري

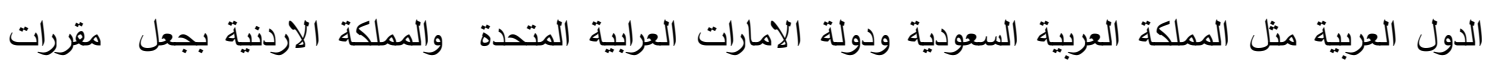
المدارس ترسل عبر الإنترنت، ولزيادة نجاح الطلاب في المقررات، تقدم لهم تثكيلة متتوعة من المصادر القائمة

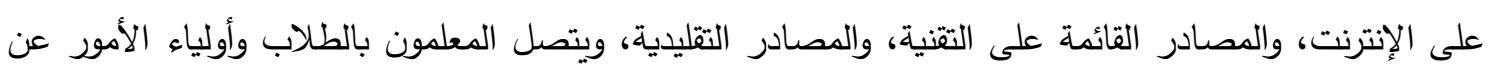
طريق البريد العادي، والهاتف، والبريد الإلكتروني ومجموعات المحادثة عبر الإنترنت.

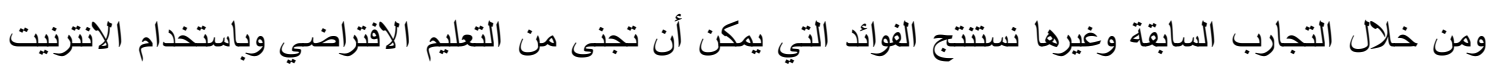

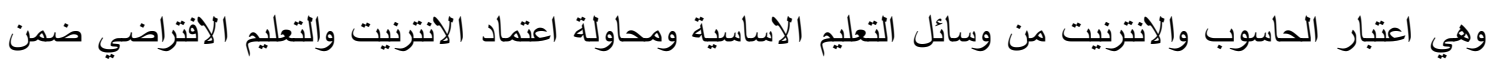

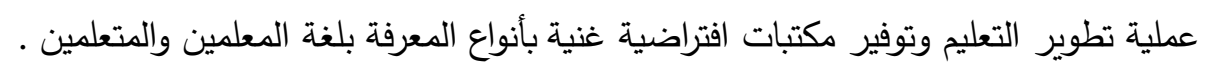

6. البرنامج التعليمي (جغرافية للصف الاول المتوسط Geography) من خلال الاطلاع على تجارب الدول العربية والعالمية انطلقت فكرة البحث بتصميم مدرسة افتراضية (موقع إلكتروني) يخدم القطاع التعليمي بالدرجة الأولى ، ويكون هذا الموقع مرتبطاً بشبكة الإنترنت حيث يمكن

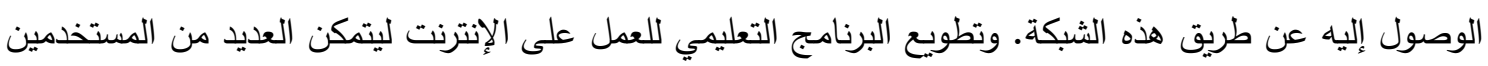

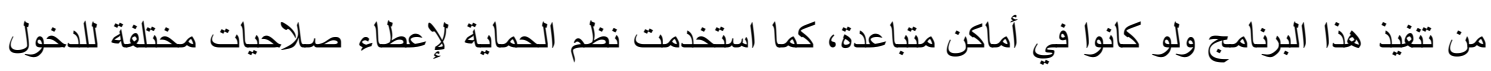

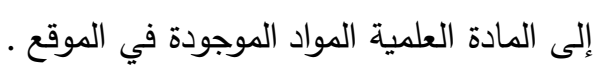

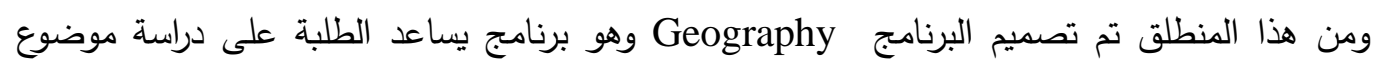

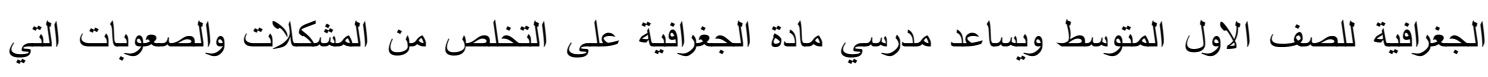

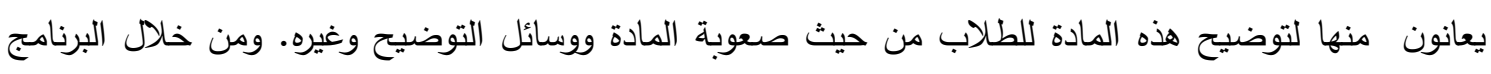

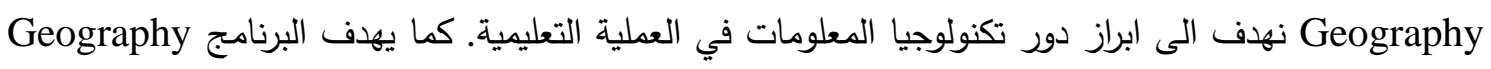


الى توفير برنامج تعليمي حيث يمكن الاستفادة منه داخل الفصل وخارجه، كما يساعد البرنامج المصمم على التواصل بين مختلف فئات القطاع التعليمي (الطالب والمعلم والمشرف) من خلال البريد الإكتروني وربط الطالب لئل بالمدرسة خارج اوقات الدوام.ويساعد ايضا في نشر الثقافة الحاسوبية من خلال تشجيع القطاع التعليمي لاقتتاء الحاسوب واستخدامه كي يتمكنوا من استخدام البرنامج التعليمي المنشور على شبكة الانترنيت.

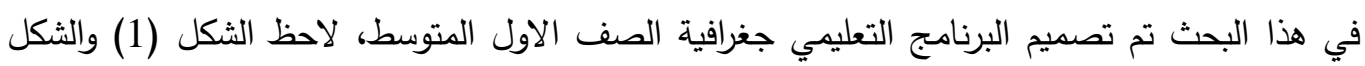

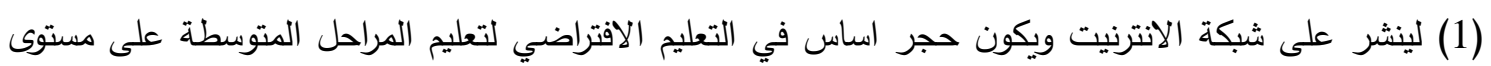

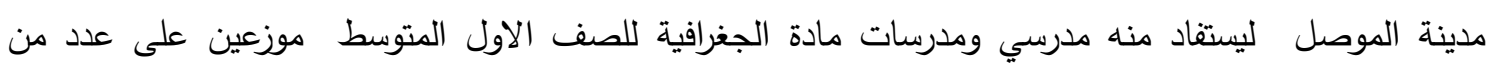

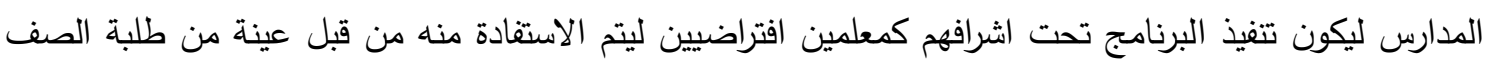
الاول المتوسط بعدر 13-14 سنة في عدد من المدارس المتوسطة المختلفة في مدينة الموصل وتم تطبيق البرنامج المصدم على هذه العينة من الطلبة والمدرسين

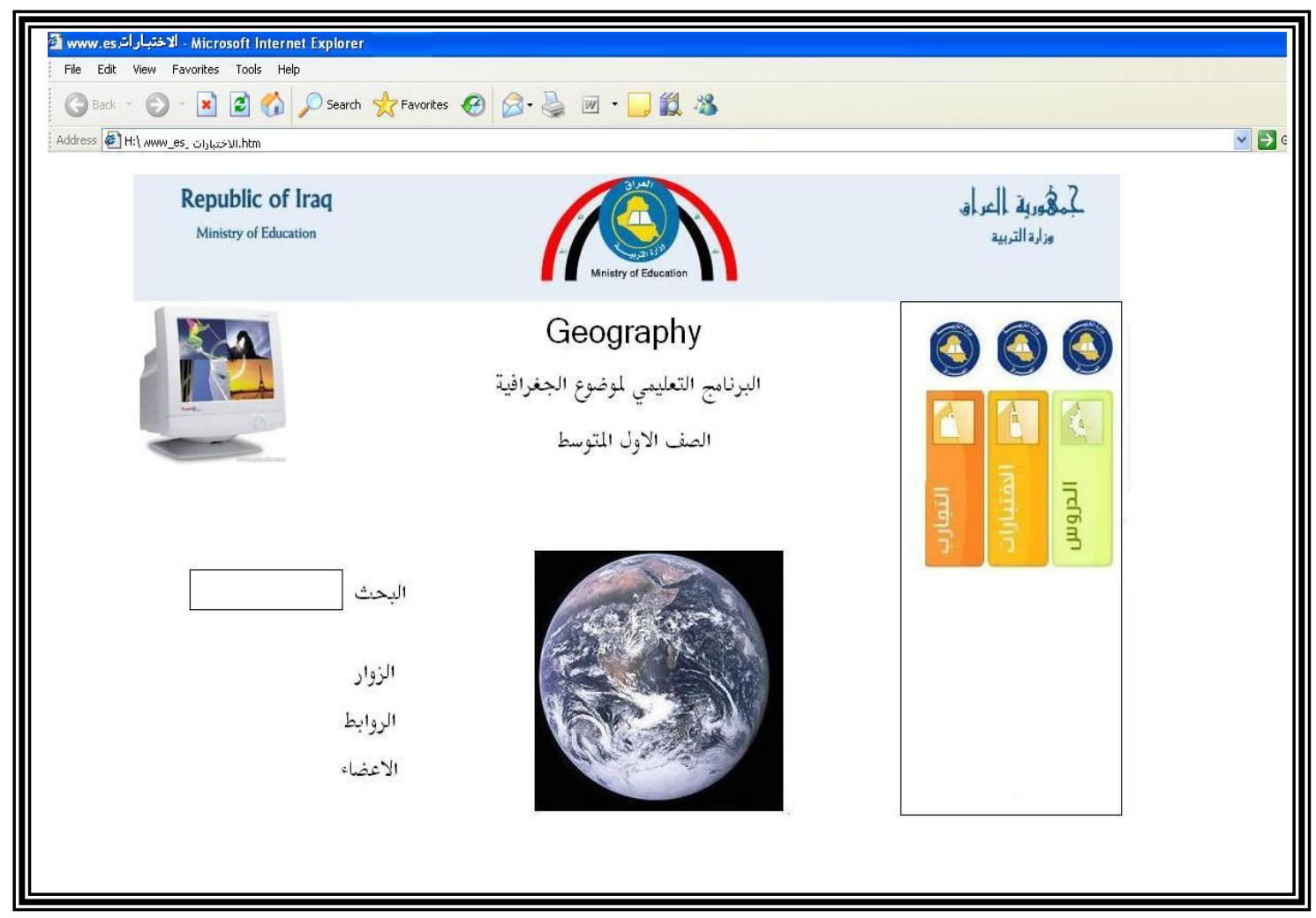

الثكل (1) الصفحة الرئيسية للبرنامج التعليمي الافتراضي (جغرافية للصف الاول المتوسط) 


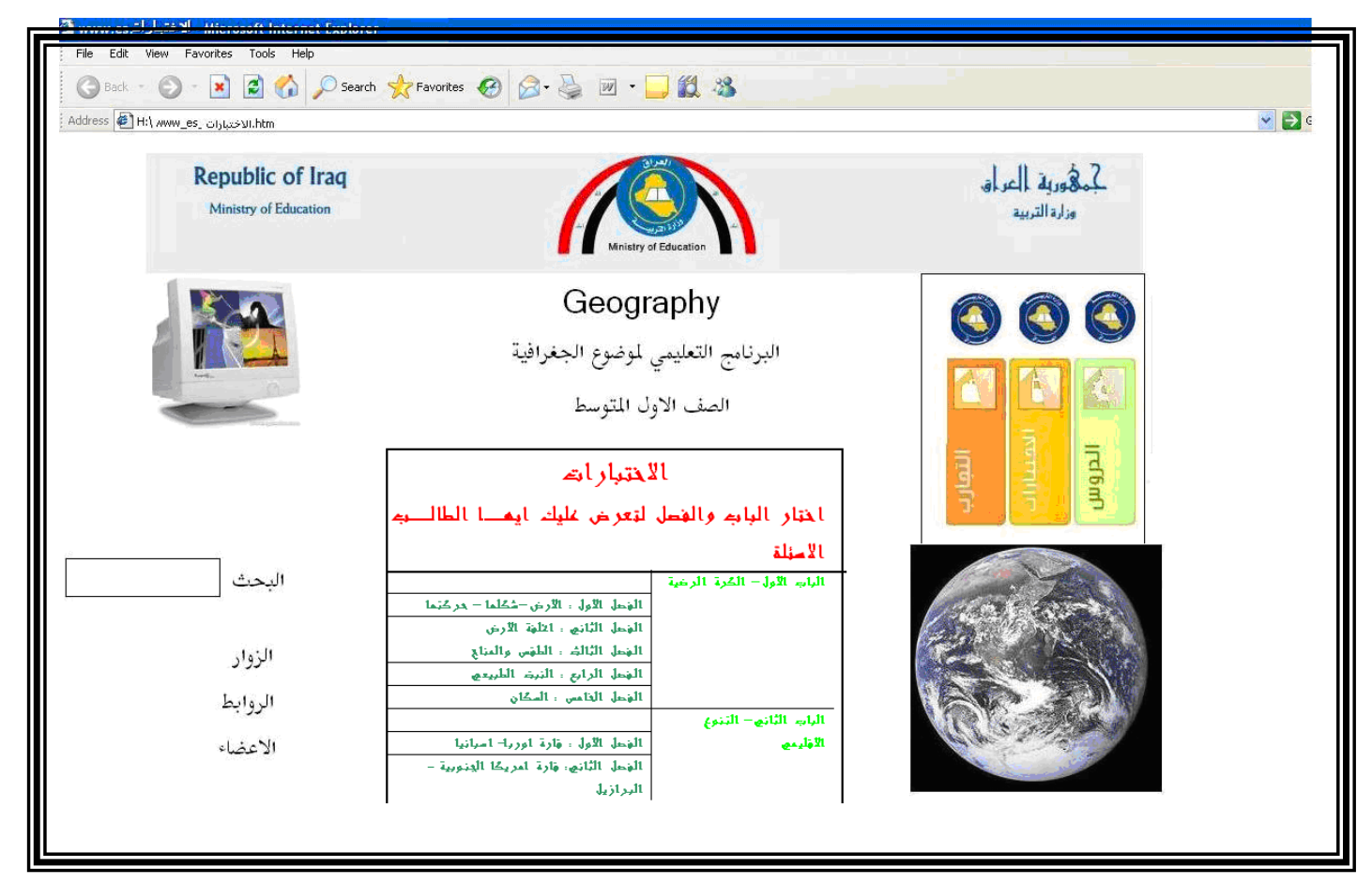

الثكل (2) صفحة الاختبارات للبرنامج التعليمي الافتراضي (جغرافية للصف الاول المتوسط)

6.1

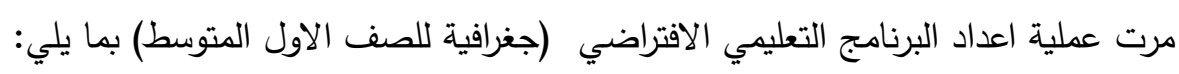

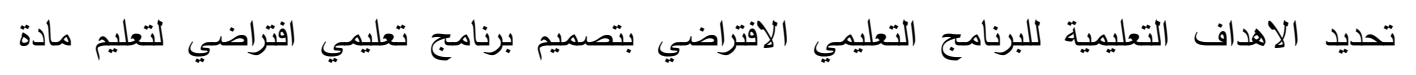

$$
\text { الجغرافية للصف الاول المتوسط. }
$$

$$
\text { تحديد مستوى المتعلمين (الصف الاول المتوسط). }
$$

تحديد المادة التعليمية وهي تعليم الجغرافية.

تحديد نظام عرض المادة التعليمية للبرنامج بحيث تتدرجيم من الاسهل الى الإديه الصعب.

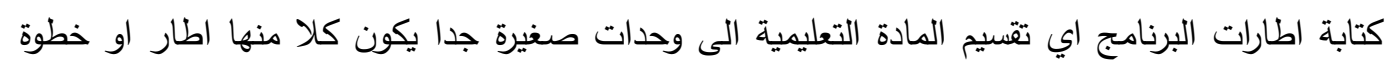

وكل اطار يتكون من المعلومات والمثيرات والاستجابات التي يتبعها التغذية الراجعة والتعزيز الفوري. تصميم موقع للبرنامج التعليمي.

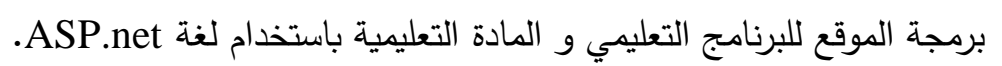

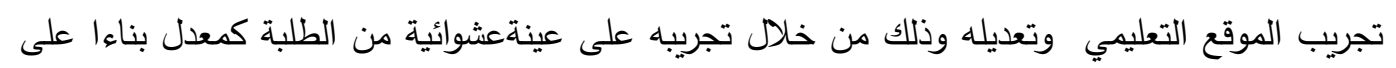
ما يحصل عليه من تغذية راجعة من الطلبة.

$$
\text { نشر الموقع على الانترنت. }
$$




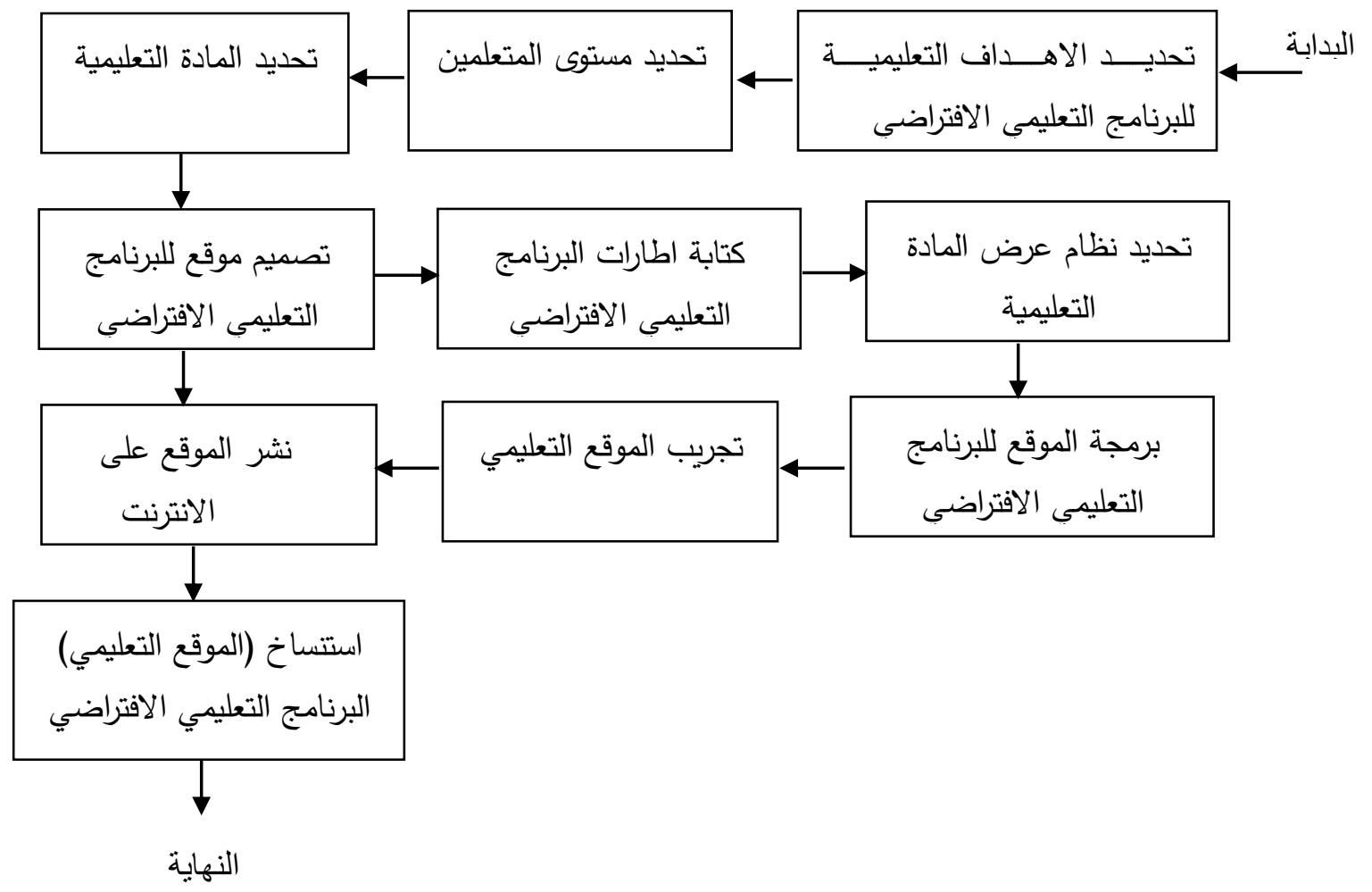

الثكل (3)مخطط يوضح المراحل الرئيسية لاعداد البرنامج التعليمي الافتراضي (الجغرافية للصف الاول المتوسط)

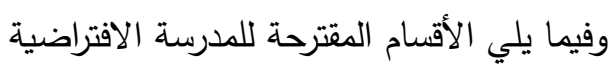
أولاً: المواد الدراسية: الجغرافية : كثرح للمادة العلمية ، روابط للمواقع ذات الصلة على الإنترنت ،كما يكون هناك مجال للطالب لطرح الأسئلة وتلقي الإجابات عليها واختبار للطلبة . ويشرف على هذا القسم العضاء الهيئة التدريسية المتخصصين بموضوع الجغرافية ثانياً: الإرشاد الطلابي: و يضم الدليل التربوي ، مشكلات وحلول ، روابط للمواقع ذات الصلة على الإنترنت،

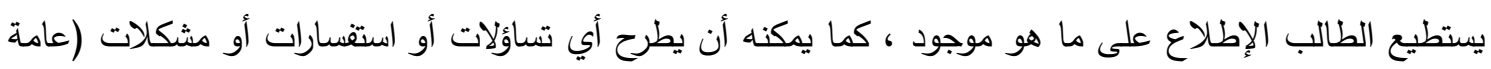

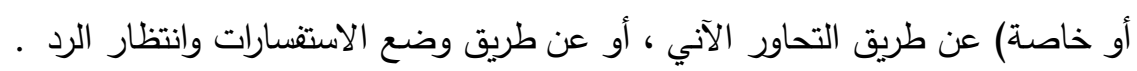

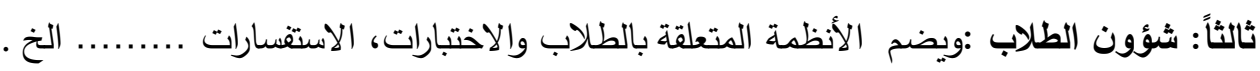

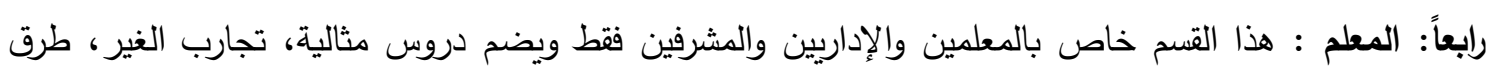

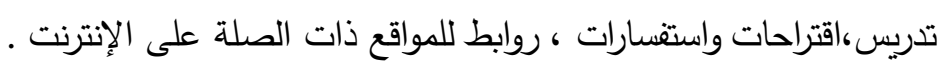

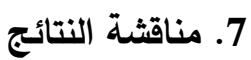

تم تتفيذ البرنامج التعليمي لمادة الجغرافية للصف الاول المتوسط من قبل عينة من مدرسي وطلاب

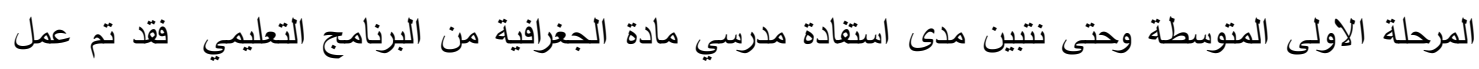

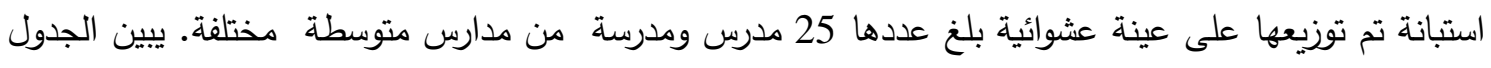


دلرسة مقارنة بين التعليم التقليلي والتعليم الافتراضي (تصميم نظام تعليمي افتراضي)

\begin{tabular}{|c|c|c|c|}
\hline النسبة الى العدد الكلي & 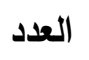 & 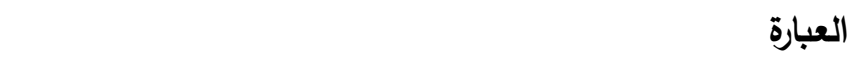 & ت \\
\hline$\% 84$ & 21 & يؤيدون استخدام التعليم الافتراضي في العملية التعليمية & 1 \\
\hline$\% 16$ & 4 & لا يؤيدون استخدام التعليم الافتراضي في العملية التعليمية & 2 \\
\hline$\% 88$ & 22 & يؤيدون استخدام الحاسوب في العملية التعليمية خارج الصف & 3 \\
\hline$\% 88$ & 22 & يؤيدون استخدام الانترنيت في العملية التعليمية خارج الصف & 4 \\
\hline$\% 76$ & 19 & يمتلكون جهاز حاسوب من اعضاء الهيئة التدريسية & 5 \\
\hline$\% 84$ & 21 & يعتقدون ان التعامل مع الحاسوب غير صعب & 6 \\
\hline$\% 60$ & 15 & يمتلكون الاتصال مع شبكة الانترنيت من اعضاء الهيئة التدريسية & 7 \\
\hline$\% 84$ & 21 & يعتقدون ان التعامل مع الانترنيت غير صعب & 8 \\
\hline
\end{tabular}

\section{جدول (1) نتائج استبانة اعضاء الهيئة التدريسية لمادة الجغرافية}

نلاحظ ان نتائج الاستبانة مشجعة وإيجابية بثكل عام فيما يخص الاستفادة من الحاسوب والانترنيت في العملية

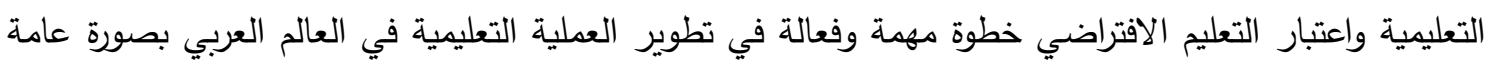
والعراق بصورة خاصة. ولنتبين موقف الطلاب فقد تم عمل استبانة لطلاب المرحلة المتوسطة، وزعت على 100 طالب من عشر مدارس. يبين الجدول (2) أهم نتائجها. وكما يتضح من الجدول ، فإن النتائج في صالح التعليم الافتراضي في العملية التعليمية.

\begin{tabular}{|c|c|c|c|}
\hline النسبة الى العدد الكلي & 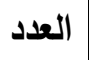 & 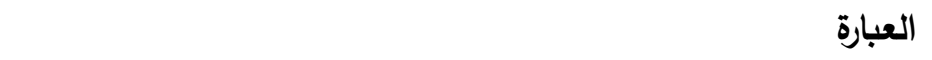 & ت \\
\hline$\% 85$ & 85 & ل لديهم اجهزة حاسوب في المنزل & 1 \\
\hline$\% 73$ & 73 & لديهح خدمة شبكة الانترنيت & 2 \\
\hline$\% 91$ & 91 & يعتقد ان استخدام الحاسوب داخل الصف سيسهل العمليةالتعليمية & 3 \\
\hline$\% 91$ & 91 & يعتقد ان استخدام الانترنيت داخل الصف سيسهل العمليةالتعليمية & 4 \\
\hline$\% 80$ & 80 & يعتقد ان استخدام الحاسوب خارج الصف سيسهل العمليةالتعليمية & 5 \\
\hline$\% 80$ & 80 & يعتقد ان استخدام الانترنيت خارج الصف سيسهل العمليةالتعليمية & 6 \\
\hline$\% 93$ & 93 & يعتقدون ان التعامل مع الحاسوب غير صعب & 7 \\
\hline$\% 92$ & 92 & يعتقد ان التعليم الافتراضي (البرنامج التعليمي لمادة الجغرافية)مفيد & 8 \\
\hline$\% 8$ & 8 & يعتقد ان التعليم الافتراضي (البرنامج التعليمي لمادة الجغرافية) غيرمفيد & 9 \\
\hline
\end{tabular}

جدول (2) نتائج استبانة الطلاب 
من خلال الاطلاع على تجارب دول العالم والدول العربية تم الاستتتاج بان المجموعات التجريبية (التي درست باستخدام الحاسوب والانترنيت (التعليم الافتراضي) ) قد تفوقت على المجموعات التي لم تستخدم فكرة التعليم الافتراضي. كما توصلت نتائج الاستبيان الموضحة في الجدول (1)و(2) حول التعليم الافتراضي في تدريس مادة الجغرافية وتصميم مجتمع تعليمي افتراضي والتي تؤيد ادخال التعليم الافتراضي للعملية التعليمية من اعضاء الهيئة

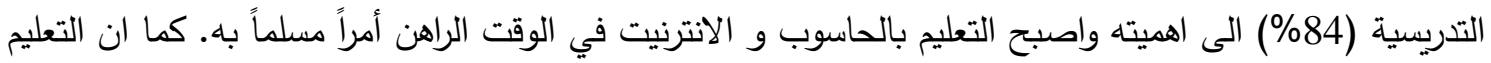
الافتراضي وفر المساندة لاعضاء الهيئة التدريسية بايجاد نوع من التوازن في توصيل المعلومات للطلاب للتخلص

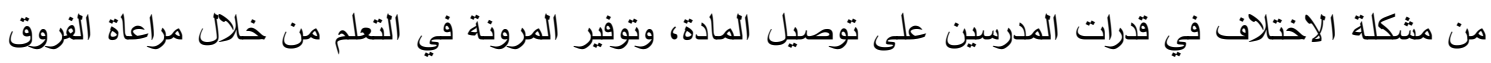

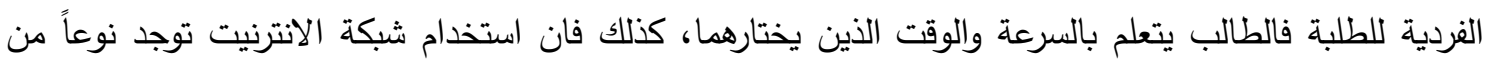
التوحيد في الموضوعات التي يراد إيصالها للطلاب وذلك من خلال توحيد مصدر المعلومات، حل مشكلات

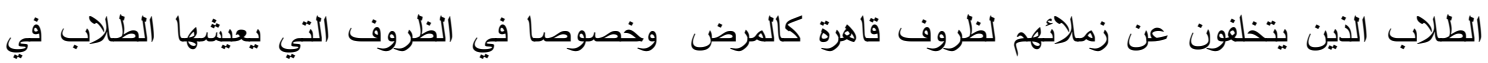

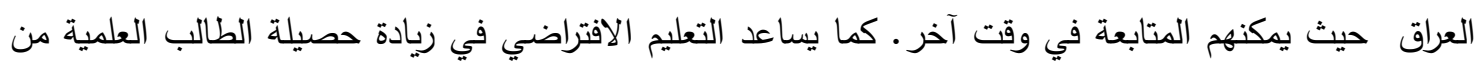

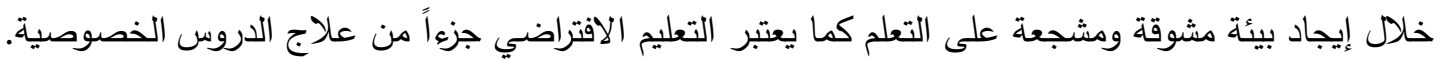

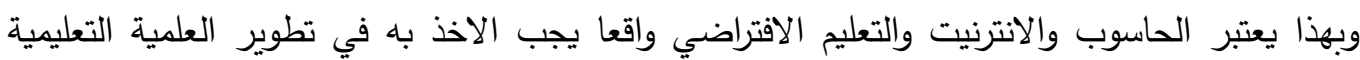

$$
\text { على مستوى العراق. }
$$

9. - الاعمال المستقبلية

تصميم مدرسة الكترونية وتحديد الاعتماد النظامي لشهاداتها. 


\section{المصادر}

ابراهيم المحيسن. 1996 ، "المعلوماتية في التعليم"، مجلة عربيوتر ، عدد 73.

ابراهيم المحيسن • 1423هـ ، "التعليم الاككتروني ترف ام ضرورة"، ورقة عمل مقدمة الى الندوة العلمية

$$
\text { (مدرسة المستقبل)، جامعة الملك سعود. المين. }
$$

حورية المالكي. 2001 م ، "الإنترنت في العملية التعليمية"، وزارة التربية والتعليم والتعليم العالي، الدوحة

حيدر طالب الأحمر ، التعليم الاكتروني... حاجة أم وجاهة، مركز الفرات للتمية والدراسات

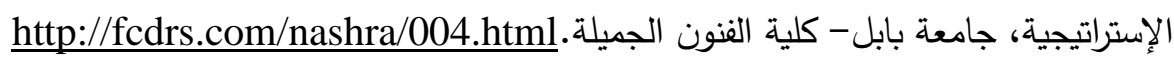

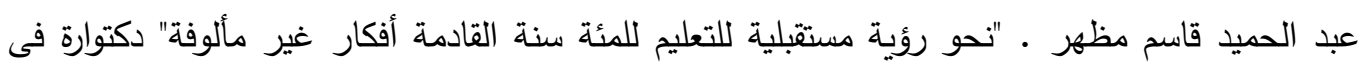

هندسة وعلوم الطيران والفضاء من معهد جورجيا للتكنولوجيا، أتلانتا، ولاية جورجيا، الولايات المتحدة

عبد القادر الفنتوخ ومهندس عبد العزيز السلطان. 1999م، " الإنترنت في التعليم : مشروع المدرسة

الإلكترونية" ، رسالة الخليج العربي ، الرياض ،عدئ لعدد 71.

عبد الله سعد العمري. 2001 م ،" تكنولوجيا الحاسوب في العملية التعليمية" ،مجلة دراسات في المناهج

وطرق التدريس ، العدد الثالث والسبعون ، مصر - القاهرة .

مصطفى بن محمد عيسى فلاته 1416.هـ، " المدخل إلى التقنيات الحديثة في الاتصال و التعليم".

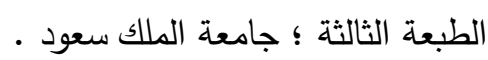

$$
\text { هشام محمد الحرك. "الإنترنت في التعليم : مشروع المدرسة الإلكترونية" }
$$

[10] Swadi H., Vertual Iraq University, http://www.annabaa.org/nbahome/nba70/ivu.htm

[11] http://www.eschool.gov.sa/index.php?option=com_content\&task=view\&id=38 \&Itemid=33

[12] http://www.khayma.com/education-technology/in3.htm

[13] http://www.flvs.net 